\title{
A Comparison between Fuzzy Inference Systems for Prediction (with Application to Prices of Fund in Egypt)
}

\author{
Raafat Fahmy \\ Lecture of Statistics, Dept. of \\ Statistics and Mathematics, \\ Benha University, Egypt
}

\author{
Hegazy Zaher \\ Professor of Mathematical \\ Statistics, Institute of Statistical \\ Studies and Research (ISSR), \\ Cairo University, Egypt
}

\author{
Abd Elfattah Kandil \\ Professor of Statistics, Dept. of \\ Statistics and Mathematics, \\ Benha University, Egypt
}

\begin{abstract}
This paper outlines the basic differences between the Fuzzy logic techniques, including Mamdani, Sugeno fuzzy inference system models and Adaptive Neuro-Fuzzy Inference System (ANFIS). The main motivation behind this research is to assess which approach provides the best performance for predicting prices of Fund. Due to the importance of performance in Economy, the Mamdani, Sugeno models and ANFIS are compared with the actual values. Fuzzy inference systems (Mamdani, Sugeno and ANFIS fuzzy models ) can be used to predict the weekly prices of Fund for the Egyptian Market. The application results indicate that (ANFIS) model is better than that of Mamdani and Sugeno. The results of the three fuzzy inference systems (FIS) are compared.
\end{abstract}

\section{Keywords}

Prices of Fund, ANFIS model, Fuzzy Inference System (FIS), Fuzzy Logic, Mamdani model, Sugeno model.

\section{INTRODUCTION}

Due to the ever-changing economic environment, it is noticed that the change happens in many periods measured by years, months, weeks and days. However, it so happens that a sudden change occurs, such as the recent world economic crisis that has had its serious consequences. Thus, the importance of building models for forecasting lies in the expectation of such crises. The Fuzzy logic is closer in spirit to human thinking and natural language than conventional logical systems are. The Fuzzy Logic method is a relatively modern method. It depends on the obscurity logic which is one of the ways of logic.

An adaptive Neuro-Fuzzy inference (ANFIS) system, which was used for rainfall-runoff modeling for the Nagwan watershed in the Hazaribagh District of Jharkhand, India, was used by Kumar [1]. Bireka [2] developed a fuzzy logic-based approach to leakage forecasting in the water industry. Alvisi [3] showed water level forecasted through Fuzzy Logic and Artificial Neural Network Approaches. Aqil [4] used A Takagi-Sugeno Fuzzy System for the Prediction of River Stage Dynamicsmes. Keskin [5] applied Fuzzy Logic approaches to flow Predicted Dim Stream. Mahabir [6] applied Fuzzy Logic for Forecasting Ice Jam Risk at Fort Mcmurray. A comparative study of statistical and neuro-fuzzy network models for forecasting the weather of Goztepe, Istanbul, Turkey, was presented by Tekta [7] A time series prediction model for daylight interior illuminance obtained using Adaptive neuro fuzzy inference system (ANFIS) was presented by Kurian [8].

A fuzzy logic-based system to predict bankruptcy for one, two and three years before the possible failure of companies was used by Korol [9]. Sajfert [10] exemplified the possibility of applying fuzzy logic into the process of decision making regarding the selection of executive managers. Reliable prediction of sales can improve the quality of business strategy by Chang [11]. Fuzzy approach for risk evaluating and forecasting in accidents caused by working with vehicles such as lift truck was used by Naieni [12]. Pasila [13] used Neuro-Fuzzy Approaches for Forecasting Electrical Load. A new peak power optimization algorithm of the electric energy consumption with nonlinear prediction is presented by Dankovi'c [14]. Ferreira [15] aimed to develop an algorithm using fuzzy sets to predict the estrus in dairy cows. Zaher [16] compared Mamdani and Sugeno Fuzzy Inference Systems for Prediction (with Application to Prices of Fund in Egypt). Kaur [17] shows that Sugeno results for air conditioning system are relatively better than those of Mamdani.

From previous studies, it is observed that there are many studies that have used fuzzy logic in various fields. However, no comparison was found between (ANFIS), Mamdani and Sugeno fuzzy inference system in predicating investment fund prices. This Paper provides a comparison between (ANFIS), Mamdani and Sugeno fuzzy inference systems in predicting investment fund prices

\section{MAMDANI FIS VS SUGENO FIS}

In terms of the inference process, there are two main types of Fuzzy Inference Systems (FIS): the Mamdani [18] and the Sugeno type [19].

In terms of use, the Mamdani FIS is more widely used mostly because of the reasonable results with a relatively simple structure it provides, and the intuitive interpretable nature of the rule base [20]. Since the consequents of the rules in a Sugeno FIS are not fuzzy, this interpretability is lost; however, the Sugeno FIS's rules' consequents can have as many parameters per rule as input values, which results in more degrees of freedom in the design than those of Mamdani and, in turn, provides the system's designer with more flexibility in the design of the system [21].

In many decision support applications, it is important to guarantee the expressive power, easy formalization and interpretability of Mamdani-type fuzzy inference systems (FIS) while ensuring the computational efficiency and accuracy of Sugeno-type FIS [22]. Hence, the fact that a Mamdani FIS can be seen as a function that maps the system's input space into its output space ensures that there exists a Sugeno FIS that can approximate any given Mamdani FIS with an arbitrary level of precision. It is beyond the scope in this paper to explain in detail the formalisms of this comparison. For a comprehensive comparison and description on several approximate reasoning methods, including Mamdani FISs and Sugeno FISs, see [23]. To sum up, the 
main motivations for testing the classification developed with the Mamdani/Sugeno FIS and comparing the results are:

1. The Sugeno FIS is more flexible because it allows more parameters in the output. Since the output is a function of the inputs, it expresses a more explicit relation among them;

2. In computational terms, the Sugeno FIS is more effective because the complex defuzzification process of the Mamdani FIS is replaced with a weighted average;

3. Because of the structure of the Sugeno FIS rule outputs, it is more convenient for functional analysis than a Mamdani FIS is.

From the above-mentioned results, it seems that any Sugeno FIS is always more efficient than a Mamdani FIS. In conclusion, in this research only the "generic system level alarms" module is considered for performing the comparison.

\section{ANFIS MODEL}

The acronym ANFIS derives its name from adaptive neurofuzzy inference system. Using a given input/ output data set, the toolbox function ANFIS constructs a fuzzy inference system (FIS) whose membership function parameters are tuned (adjusted) using either a back propagation algorithm alone or in combination with the least squares type of method. This adjustment allows your fuzzy systems to learn from the data they are modeling. See [24]

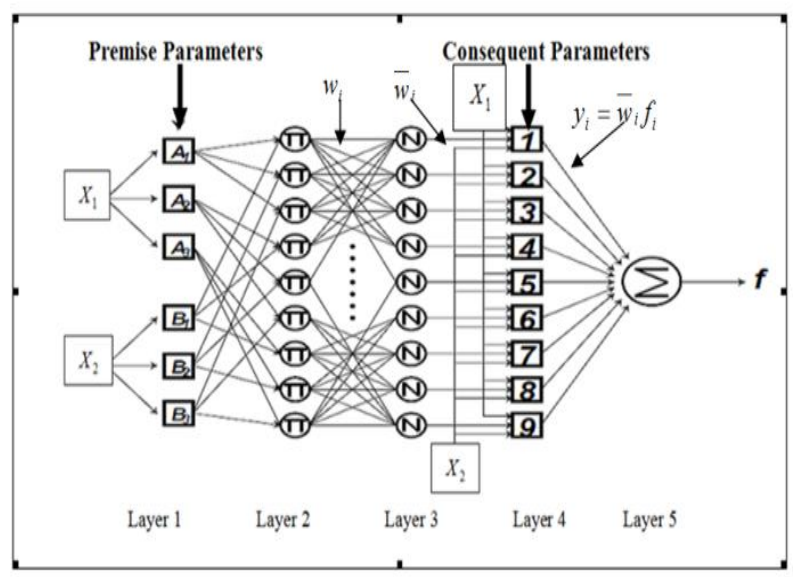

Fig. 1 Explain ANFIS Structure

The ANFIS approach defines a Takagi-Sugeno fuzzy inference system through a Neural Network approach by defining 5 layers:

- Layer 1: fuzzyfication of the input values due to MSFs -> membership degrees.

$$
O_{i}^{1}=\mu_{A_{i}}\left(x_{1}\right), \mu_{B_{i}}\left(x_{2}\right)
$$

where $X_{1}$ and $X_{2}$ are the inputs.

- Layer 2: aggregation of membership degrees due to an appropriate t-norm applied in the premise parts.

$$
O_{i}^{2}=w_{i}=A N D \operatorname{rule}\left(\mu_{A_{i}}\left(x_{1}\right), \mu_{B_{i}}\left(x_{2}\right)\right)
$$

- Layer 3: evaluation of the basis functions by normalization of aggregated membership degrees.

$$
O_{i}^{3}=\overline{w_{i}}=\frac{w_{i}}{w_{1}+w_{2}+\cdots+w_{9}}
$$

- Layer 4: weighting of basis functions with linear (=> Takagi-Sugeno system) or constant (=> Sugeno system) consequent functions.

$$
O_{i}^{4}=y_{i}=\overline{w_{i}} f=\overline{w_{i}}\left(p_{i} x_{1}+q_{i} x_{2}+r_{i}\right)
$$

- Layer 5: evaluation of output values by applying

$$
O_{i}^{5}=\sum_{i} \overline{w_{i}} f_{i}
$$

\section{APPLICATIONS OF FIS}

\subsection{Shows the Application of Mamdani FIS}

Prices of fund in the Egyptian market are predicted using the Mamdani fuzzy model. It consists of one input Price levels. The system has one output that Market conditions. The prices are taken to be in the ranges of 450 to 2550 . The researcher applied the method of Mamdani gbellmf, trying to find the best one for prediction. The input has nine membership functions as shown in Fig. (2). The output (Market conditions) is taken in values in range from 450 to 2550 and have nine Triangular membership functions shown in Fig. (3). The Rule base of Mamdani-type FIS is shown in Table (1). Finally, the comparison between the actual value and Mamdani gbellmf values is shown in Fig. (4). see [16]

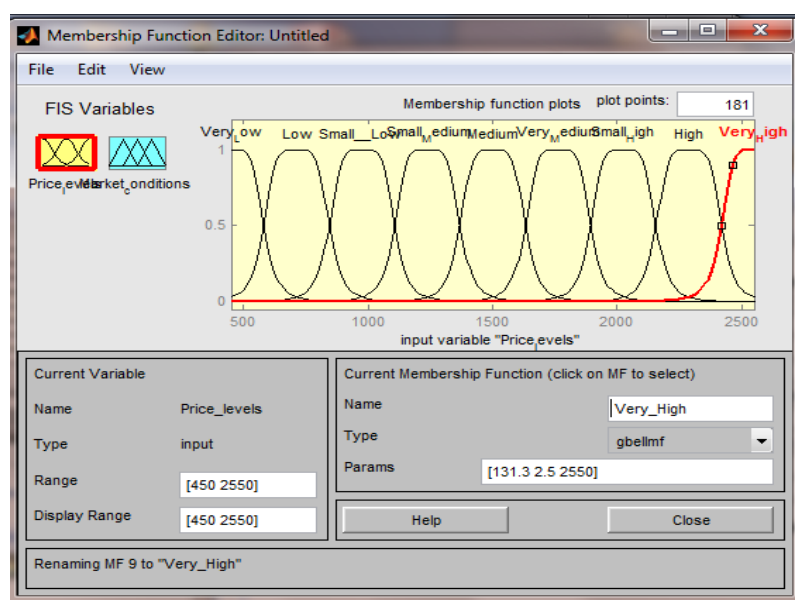

Fig. 2 Mamdani Bell membership function

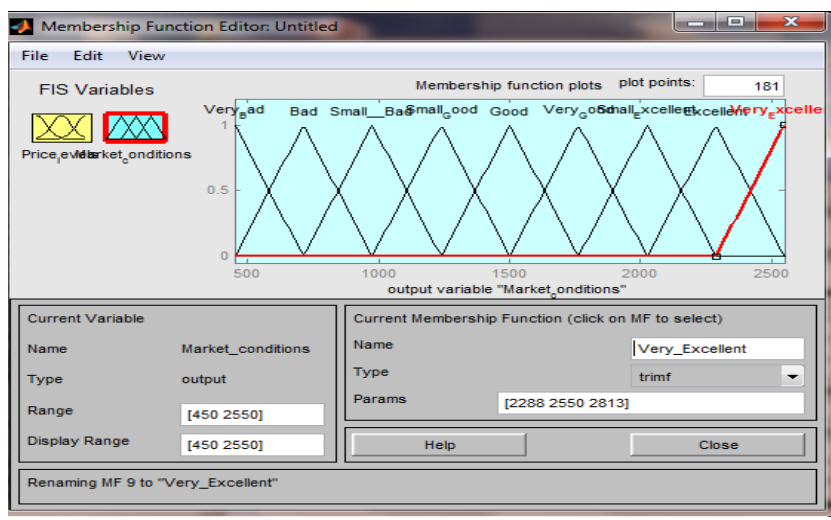

Fig. 3 Market conditions Triangular membership function. 
Table 1: Rule base of Mamdani FIS

\begin{tabular}{|c|c|c|}
\hline Rules & Price levels & $\begin{array}{c}\text { Market } \\
\text { conditions }\end{array}$ \\
\hline Rule 1 & Very Low & Very Bad \\
\hline Rule 2 & Low & Bad \\
\hline Rule 3 & Small Low & Small Bad \\
\hline Rule 4 & Small Medium & Small Good \\
\hline Rule 5 & Medium & Good \\
\hline Rule 6 & Very Medium & Very Good \\
\hline Rule 7 & Small High & Small Excellent \\
\hline Rule 8 & High & Excellent \\
\hline Rule 9 & Very High & Very Excellent \\
\hline
\end{tabular}

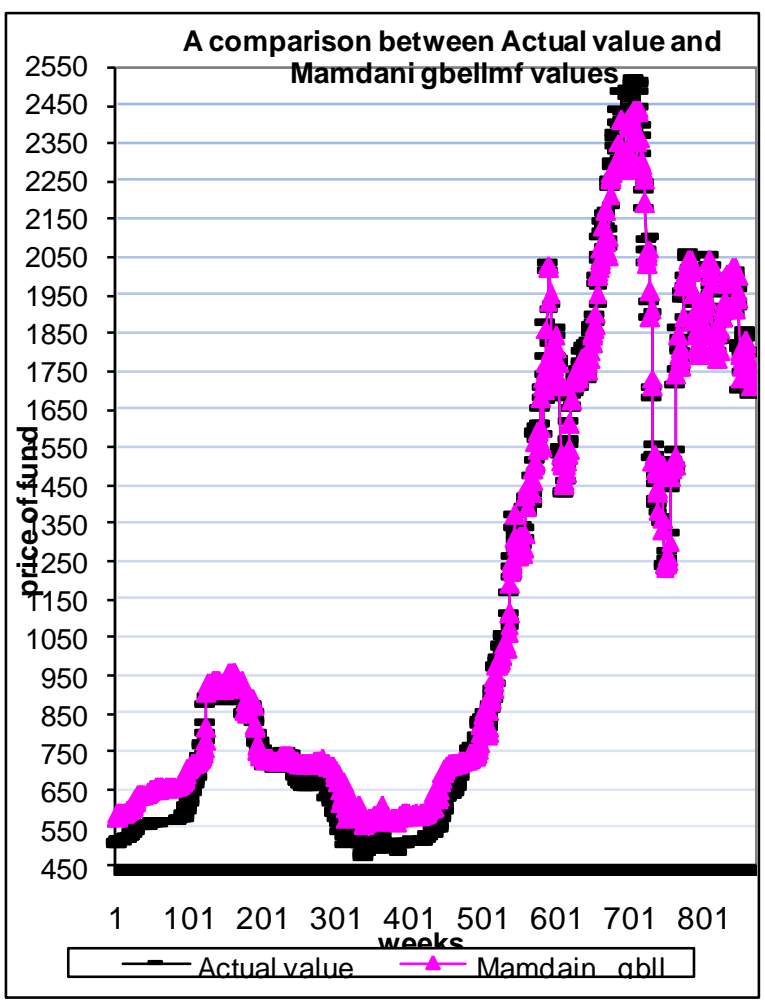

Fig. 4: showing comparison between Actual value and Mamdani gbellmf values.

\subsection{Shows the Application of Sugeno FIS}

Prices of fund in the Egyptian market are predicted using the Sugeno fuzzy model. It consists of one input Price levels. The system has one output: Market conditions. The prices are taken to be in ranges of 450 to 2550 . The researcher applied the method of Sugeno gaussmf, trying to find the best one for prediction. The input has nine membership functions as shown in Fig. (5).The output (Market conditions) is taken in values in the range from 450 to 2550 and have nine Triangular membership functions shown in Fig. (6). The Rule base of
Sugeno -type FIS is shown in Table (2). Finally, a comparison between the actual values and Sugeno gaussmf values is shown in Fig. (7). see [16]

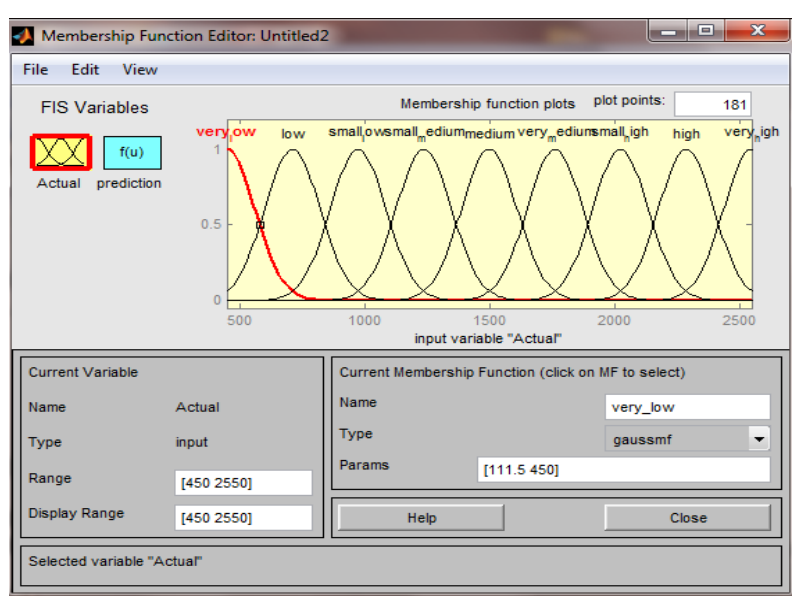

Fig. 5 Sugeno gaussmf

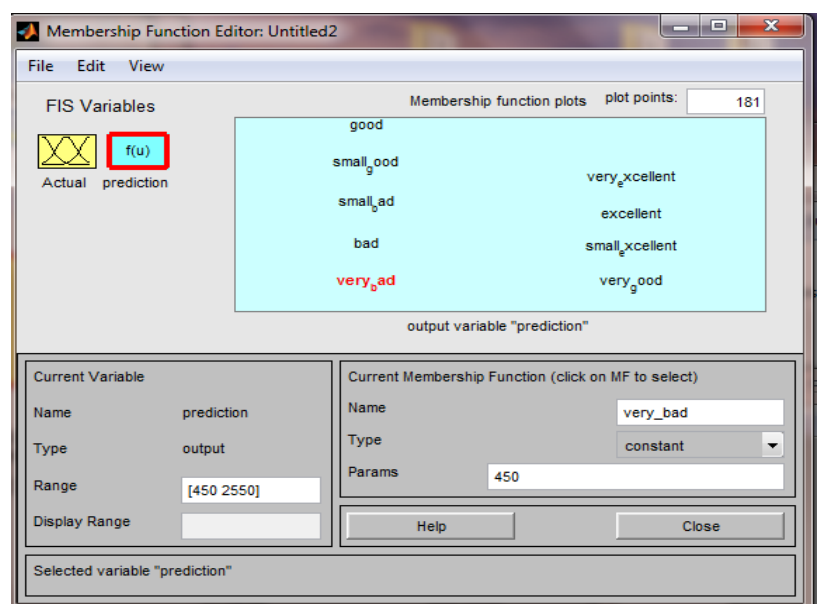

Fig. 6 The output Market conditions are taken in values in range from 450 to 2550

Table 2 : Market conditions membership functions

\begin{tabular}{|c|c|}
\hline Market conditions & Constant value \\
\hline Very Bad & 450 \\
\hline Bad & 700 \\
\hline Small Bad & 900 \\
\hline Small Good & 1300 \\
\hline Good & 1500 \\
\hline Very Good & 1750 \\
\hline Small Excellent & 2000 \\
\hline Excellent & 2250 \\
\hline Very Excellent & 2550 \\
\hline
\end{tabular}




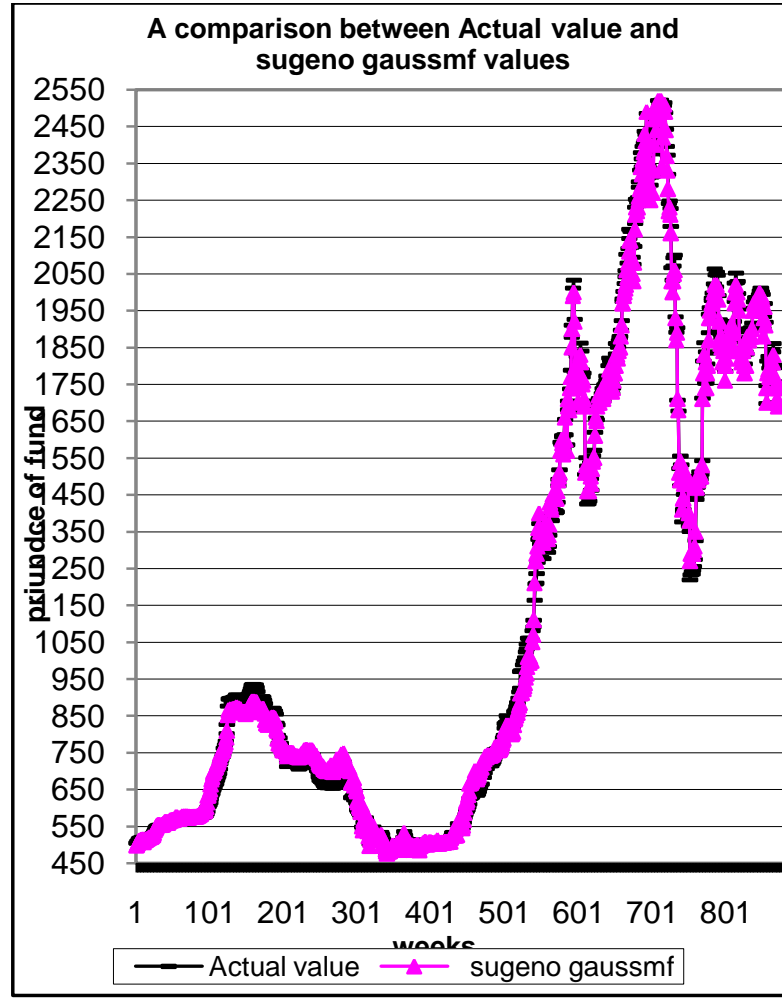

Fig. 7: showing comparison between Actual value and Sugeno gaussmf values.

\subsection{Shows the Application of ANFIS Model}

The researchers design a program by using MATLAB's programming to evaluate the ANFIS Model in the prediction. In addition to this, several attempts are conducted to reduce the rate of (RMSE) for the price of the fund. First, five membership functions and a hundred epochs were chosen, which resulted in $($ RMSE $)=139.5$. In the second attempt, the number of membership functions was 10 and the number of epochs was 200, which resulted in $($ RMSE) $=110.5$. In the third attempt, the membership functions was 15 and the number of epochs was 200, which resulted in (RMSE) $=$ 175.6. In the fourth attempt, the number of membership functions was 20 and the number of epochs was 300, which resulted in $($ RMSE $)=12.05$. In the fifth attempt, the number of membership functions was 20 and the number of epochs was 500, which resulted in (RMSE) $=84.95$. It is noticed that the fourth attempt is the best one of the results since the measure $(\mathrm{RMSE})=12.05$, which is the smallest one of them. The results of the fourth attempt were shown in Fig. 8 and Fig. 9.

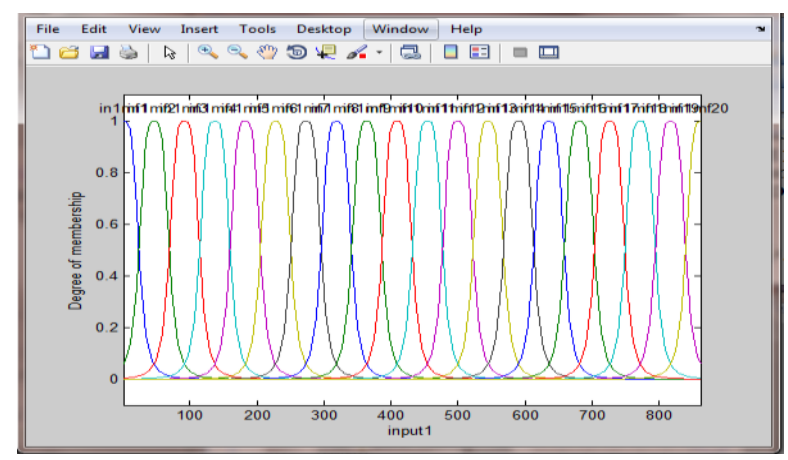

Fig. 8 Gaussian shape of the membership function

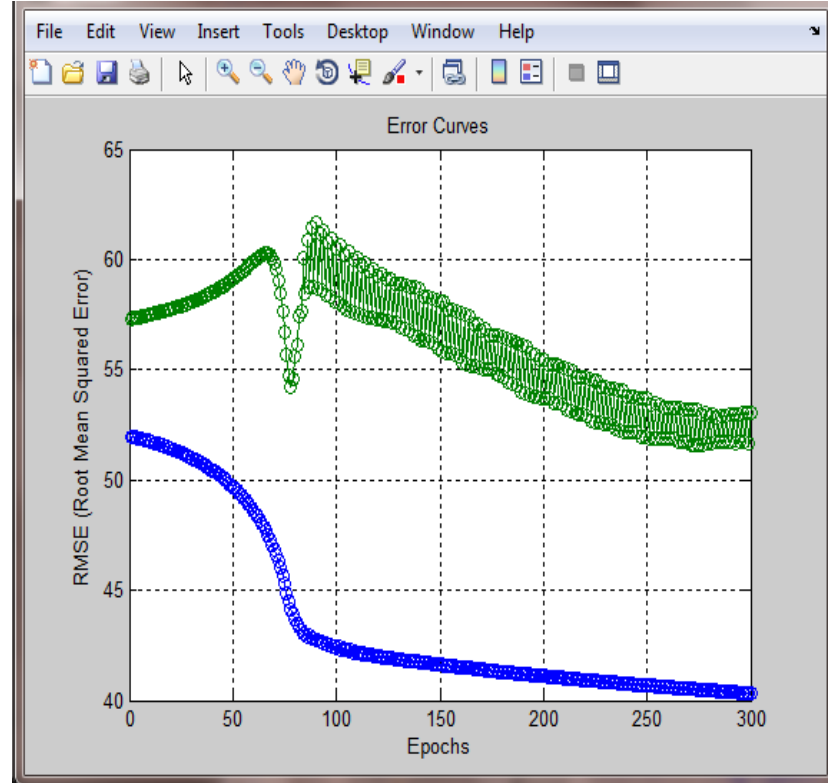

Fig. 9 (RMSE) Root Mean Squared Error of ANFIS Model

The program used and the process of prediction.

The program used was MATLAB which was used in the ANFIS model. The best results predicted were 1-862 price of fund. Actual values and ANFIS values related to the fund prediction were shown in Fig. 10

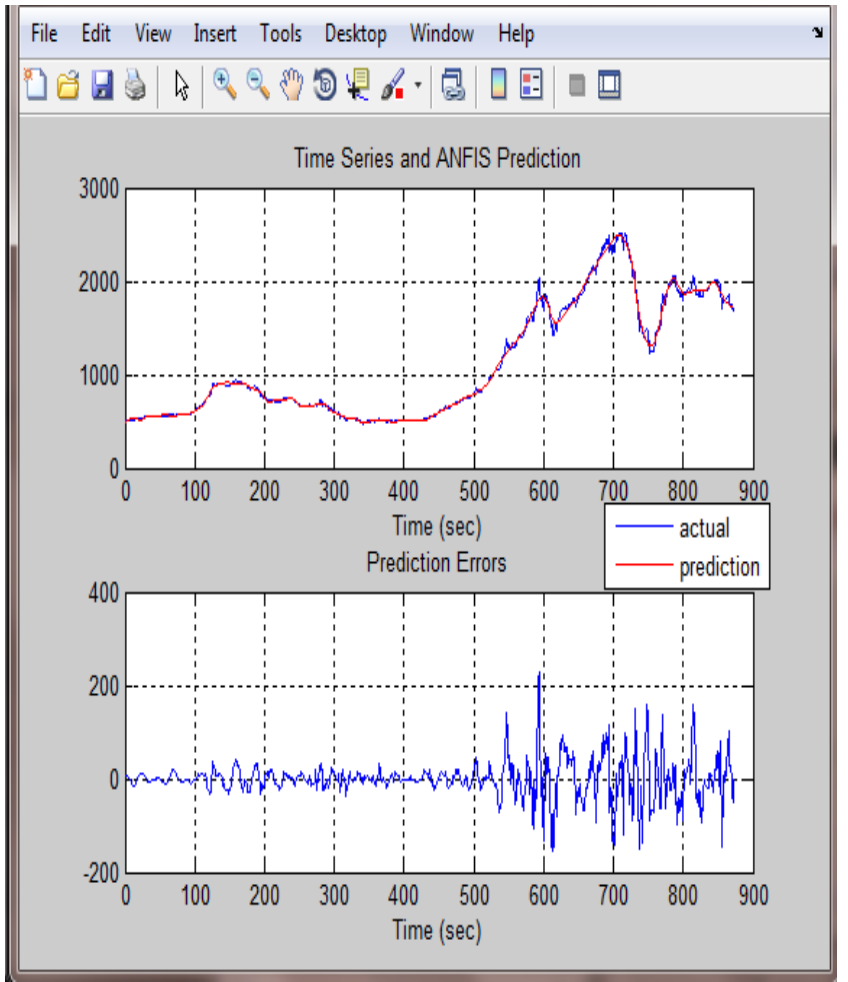

Fig. 10 time series and ANFIS prediction prices of fund per a week 


\section{RESULTS AND DISCUSSIONS}

\subsection{A comparison T-test between Actual} values $\left(\mu_{n}\right)$ and predicted values of ANFIS,

Mamdani and sugeno $X_{n}$ which is based on $(30,100,300,500$ and 862$)$ weeks as sample sizes.

$H_{0}: \mu_{n}=X_{n}$ against $\quad H_{1}: \mu_{n} \neq X_{n}$

- $\mu_{n}=X_{n}$ : There is no difference between the means

$P$-value $\succ 0.01$ It is not significant.

- $\mu_{n} \neq X_{n}$ : There is difference between the means. $P-$ value $\prec 0.01$ It is significant.

Table 3: A Comparison between the actual values (Arithmetic means $\mu_{n}$ ), and prediction values for each of the methods of ANFIS, Mamdani and Sugeno (Arithmetic means $X_{n}$ ) by T-test.

\begin{tabular}{|c|c|c|c||}
\hline $\begin{array}{c}\text { Methods Actual } \\
\text { values } \boldsymbol{\mu}_{n}\end{array}$ & ANFIS & $\begin{array}{c}\text { Mamdani } \\
\text { gbellmf }\end{array}$ & $\begin{array}{c}\text { Sugeno } \\
\text { gaussmf }\end{array}$ \\
\hline $\begin{array}{c}\text { Actual values } \\
\mathrm{n}=30\end{array}$ & $\mathrm{P}=0.617$ & $\mathrm{P}=0.000^{* *}$ & $\mathrm{P}=0.262$ \\
\hline $\begin{array}{c}\text { Actual values } \\
\mathrm{n}=100\end{array}$ & $\mathrm{P}=0.904$ & $\mathrm{P}=0.000^{* *}$ & $\mathrm{P}=0.185$ \\
\hline $\begin{array}{c}\text { Actual values } \\
\mathrm{n}=300\end{array}$ & $\mathrm{P}=0.986$ & $\mathrm{P}=0.000^{* *}$ & $\mathrm{P}=0.459$ \\
\hline $\begin{array}{c}\text { Actual values } \\
\mathrm{n}=500\end{array}$ & $\mathrm{P}=0.985$ & $\mathrm{P}=0.000^{* *}$ & $\mathrm{P}=0.935$ \\
\hline $\begin{array}{c}\text { Actual values } \\
\mathrm{n}=862\end{array}$ & $\mathrm{P}=0.996$ & $\mathrm{P}=0.016^{*}$ & $\mathrm{P}=0.970$ \\
\hline
\end{tabular}

$* * \mathrm{P} \leq 0.01$, high significant

$* \mathrm{P} \leq 0.05$, significant

Referring to the result of Table (3), it should be noticed that:

The T-test of difference between actual values and predicted values of Mamdani method of $(30,100,300,500$ and 862) weeks is significant at level P-values $<0.01$ and 0.05 . However, the T-test of difference between actual values and predicted values of both ANFIS and Sugeno methods of (100, 300,500 and 862) weeks is non-significant at level P-values > 0.01 and 0.05 .
5.2 Mean Absolute Error (MAE) for the methods of ANFIS, Mamdani and Sugeno which consist of $30,100,300,500$ and 862 weeks.

In order to be at P-values, this result can be achieved by using the Mean Absolute Error (MAE) measure as follows.

Table 4: Comparison between different methods based on MAE for the methods of ANFIS, Mamdani and sugeno

\begin{tabular}{|c|c|c|c|}
\hline MAE & ANFIS & Mamdani & Sugeno \\
\hline MAE $n=30$ & 8.2 & 70.9 & 4.7 \\
\hline MAE $n=100$ & 6.2 & 82.9 & 10.2 \\
\hline MAE $n=500$ & 11.3 & 56.7 & 21.8 \\
\hline MAE $n=862$ & 25.0 & 51.4 & 31.5 \\
\hline
\end{tabular}

Referring to the result of Table V, it should be noticed that:

The researcher found that ANFIS model and Sugeno FIS are better than Mamdani FIS. But ANFIS model is the most efficient one of them.

Finally, the researchers believed ANFIS model is very good for forecasting the price of fund in the periods of severe economic fluctuations.

\section{CONCLUSION}

This paper has examined the performance of three types of Fuzzy logic Inference systems: ANFIS, Mamdani and Sugeno for predicting prices of Fund. It also confirms that a Sugeno FIS is always more efficient than a Mamdani FIS. All in all, the performance of ANFIS method is better than that of Sugeno and Mamdani for the same fuzzy technique.

\section{REFERENCES}

[1] Kumar P. \& Kumar D. Rainfall-Runoff Modelling of a Watershed. Civil and Environmental Research. 2012; Vol. 2, pp. 35-42.

[2] Bireka L., Petrovica D. \& Boylanb J. A fuzzy Logic Based Approach to Leakage Forecasting in Water Industry. The 31st Annual International Symposium on Forecasting. 2011; pp.1-12.

[3] Alvisi S., Mascellani G., Franchini M., \& B’ardossy A. Water Level Forecasting Through Fuzzy Logic and Artificial Neural Network Approaches. Hydrology and Earth System Sciences. 2006; Vol. 10, Pp. 1-17.

[4] Aqil M., Kita I., Yano A., \& Nishiyama S. A TakagiSugeno Fuzzy System for the Prediction of River Stage Dynamicsmes. JARQ. 2006; Vol. (4), pp. 369 - 378.

[5] Keskin M., Taylan E., \& Yilmaz A. Flow Prediction Model with Fuzzy Logic approaches: Dim Stream. International Congress on River Basin Management. 2004; Pp. 439-447. 
[6] Mahabir C., Hicks F. \& Robinson F. Forecasting Ice Jam Risk at Fort Mcmurray, $\mathrm{AB}$, using fuzzy logic. International Association of Hydraulic Engineering and Research, New Zealand. 2002; 2nd-6th December, pp.112-119.

[7] Tektas M. Weather Forecasting Using ANFIS and ARIMA MODELS, A Case Study of Istanbul. Environmental Research, Engineering and Management. 2010; Vol. (51), Pp. 5 - 10.

[8] Kurian C., George V., Bhat J. \& Aithal R. ANFIS Model for the Time Series Prediction of Interior Daylight Illuminance. AIML Journal. 2006; Vol. 6, pp. 35-40.

[9] Korol T. \& Korodi A. An Evaluation of Effectiveness of fuzzy Logic Model in Predicting the Business Bankruptcy. Romanian Journal of Economic Forecasting. 2011; Vol(1), pp. 92-107.

[10] Sajfert Z., Atanasković P., Pamučar D. \& Nikolić M. Application of Fuzzy Logic into Process of Decision Making Regarding Selection of Managers. African Journal of Business Management. 2012; Vol. 6, pp. 3221-3233.

[11] Chang P. \& Wang Y. Fuzzy Delphi and BackPropagation Model for Sales Forecasting in PCB Industry. Expert Systems with Applications. 2006; Vol. 30 , pp. 715-726.

[12] Naieni S., Makui A., \& Ghousi R. An Approach for Accident Forecasting Using Fuzzy Logic Rules: A Case Mining of Lift Truck Accident Forecasting in One of the Iranian Car Manufacturers. International Journal of Industrial Engineering \& Production Research. 2012; Vol. 23, pp. 53-64.

[13] Pasila F., Ajoy K. \& Thiele G. Neuro-Fuzzy Approaches for Forecasting Electrical Load Using Additional Moving Average Window Data Filter on Takagi-Sugeno Type MISO Networks. Journal of Advanced Computational Intelligence and Intelligent Informatics. 2008; Vol.12. pp. 361-366.

[14] Dankovi'c B., Jovanovi'c Z. \& Anti'c D. Peak Power Optimization Based On Nonlinear Prediction and Fuzzy
Logic. FACTA University (NIS) SER.: ELEC. ENERG. 2005; Vol. 18, pp.431-437.

[15] Ferreira L., Yanagi-Jr T., Nããs I. \& Lopes M. Development of a Decision Making System Using Fuzzy Logic to Predict Estrus in Dairy Cows. Agricultural Engineering International: the CIGR Ejournal. 2007; Vol. IX., pp. 1-16.

[16] Zaher H., Kandil A. \& Fahmy R. Comparison of Mamdani and Sugeno Fuzzy Inference Systems for Prediction (With Application to Prices of Fund in Egypt). British Journal of Mathematics \& Computer Science. 2014; 4(21): 3014-3022, 2014.

[17] Kaur A., \& Kaur A. Comparison of mamdani-type and sugeno-type fuzzy inference systems for air conditioning system. International Journal of Soft Computing and Engineering (IJSCE). 2012;2:323-325.

[18] Mamdani EH, Assilian S. An experiment in linguistic synthesis with a fuzzy logic controller. International Journal of Man-Machine Studies. 1975; 7:1-13.

[19] Takagi T, Sugeno M. Fuzzy identification of systems and its applications to modeling and control. IEEE Trans, on Systems, Man and Cybernetics. 1985; 15:116-132.

[20] Jassbi JJ, Serra PJA, Ribeiro RA, Donati A. A comparison of mamdani and Sugeno inference systems for a space fault detection application. Automation Congress, WAC '06. World. 2006;24-26:1 - 8 .

[21] Mendel J. Uncertain rule-based fuzzy inference systems: Introduction and new directions. Prentice-Hall; 2001.

[22] Kisi O. Applicability of Mamdani and Sugeno fuzzy genetic approaches for modeling reference evapotranspiration. Journal of Hydrology. 2013;504:160-170

[23] Jassbi J, Alavi SH, Serra PJA, Ribeiro RA Transformation of a Mamdani FIS to First Order Sugeno FIS. Fuzzy Systems Conference, FUZZ-IEEE; 2007.

[24] Alavala C. Fuzzy Logic and Neural Networks Basic Concepts \& Application. New Age International (p) Ltd. New Delhi. 2009. 